\title{
Controlling lexical contributions to the reading of pseudohomophones
}

\author{
Peter J. Kwantes and Harvey H. C. Marmurek \\ University of Guelph, Guelph, Ontario, Canada
}

\begin{abstract}
Reynolds and Besner (2005) presented a computational account of six effects that emerge when readers are asked to pronounce pseudohomophones (nonwords - e.g., brane - that sound like words when pronounced). In the dual route cascaded (DRC) model of reading, they varied a parameter controlling the rate of inhibition from letter units to the orthographic lexicon to mimic strategic control over the extent of specific lexical processing. In this article, we provide an account in which the effects are simulated by varying the DRC's reading-aloud criterion-a parameter that sets the minimal level of phonemic activation required to pronounce a letter string. We show that varying this parameter provides another means of controlling lexical contributions to reading aloud.
\end{abstract}

Pseudohomophones are nonword letter strings that, when pronounced, sound like words (e.g., brane sounds like brain). Several studies (e.g., Marmurek \& Kwantes, 1996) have shown that a reader's performance when processing a pseudohomophone is influenced by properties of the letter string's base word. The nature of the influence depends on the task that a reader is asked to perform (e.g., naming or lexical decision) and the context in which the stimuli are presented (e.g., in pure lists of pseudohomophones or mixed with control nonwords that do not sound like words, such as frane).

Reynolds and Besner (2005) reviewed six phenomena that are elicited when readers name pseudohomophones aloud as quickly and as accurately as they can. Briefly, pseudohomophones are named more quickly than control nonwords (a pseudohomophone advantage) when the two classes of stimuli are mixed together in a list (Borowsky, Owen, \& Masson, 2002; Grainger, Spinelli, \& Ferrand, 2000; Herdman, LeFevre, \& Greenham, 1996; Marmurek \& Kwantes, 1996; McCann \& Besner, 1987; Taft \& Russell, 1992) but more slowly than control nonwords (a pseudohomophone disadvantage) when the two types of items are presented in separate, pure lists (Borowsky et al., 2002; Reynolds \& Besner, 2005).

In the base-word frequency effect, pseudohomophones derived from high-frequency base words are named more quickly than those derived from low-frequency base words. Whereas the base-word frequency effect is strong for pure lists of pseudohomophones, the effect is attenuated or eliminated when the pseudohomophones are mixed with control nonwords (Marmurek \& Kwantes, 1996; McCann \& Besner, 1987).

Both the pseudohomophone disadvantage and the base-word frequency effect found for pure lists are modulated by the order in which lists are presented. Specifi- cally, the pseudohomophone disadvantage and the baseword frequency effect are attenuated when the pure list of pseudohomophones is read following the reading of a pure list of control nonwords (Borowsky et al., 2002; Reynolds \& Besner, 2005).

Reynolds and Besner (2005) proposed that the pattern exhibited by pseudohomophones in the naming task reflects differing degrees to which the reader uses general and specific lexical information as strategies to help build a pronunciation for the pseudohomophone. To support this hypothesis, Reynolds and Besner simulated the two strategies in the dual route cascaded (DRC) model of word identification (Coltheart, Rastle, Perry, Langdon, \& Ziegler, 2001). The DRC model postulates that the pronunciation of a letter string is constructed using two routes, referred to as the lexical and the nonlexical routes. The architecture of the lexical route includes two lexicons: an orthographic lexicon containing the spellings of all words known to the system, and a phonological lexicon containing their pronunciations.

When a letter string is presented to the model, its characters activate letter units that, in turn, activate entries in the orthographic lexicon. Activation from the orthographic lexicon spreads to entries in the phonological lexicon. From the phonological lexicon, word entries activate nodes in a phoneme system used to hold a letter string's final pronunciation. Over successive processing cycles, the lexical route uses feed-forward connections to the phoneme system as well as feedback cascading all the way back to the letter units, to settle on a pronunciation. If the letter string exists in the lexicon and the system is given enough time, the lexical route will settle on the correct entry and the word's pronunciation in the phonological lexicon will determine the activated phonemes in the phoneme system. The nonlexical route translates a letter

H. H. C. Marmurek, marmurek@psy.uoguelph.ca 
string into a string of phonemes by applying graphemeto-phoneme translation rules to each letter, one at a time, starting with the first letter.

The two routes operate simultaneously. Some letter strings can be named via either route. Words that follow the rules governing spelling-sound correspondence (socalled "regular words"- e.g., mint) can be read correctly via either route. Other classes of letter strings can make use of only one route. Words that violate spelling-sound correspondence rules (so-called "irregular words"-e.g., pint) require the lexical route to generate a correct response. Applying spelling-sound correspondence rules to an irregular word leads to an incorrect pronunciation. By contrast, nonwords must be read via the nonlexical route because corresponding entries do not exist in the lexicons. Together, the routes converge on a pronunciation for any letter string presented to the model. The time it takes to reach a pronunciation depends on, among other things, the familiarity of the letter string and the level of agreement between the outputs of the two routes.

Reynolds and Besner (2005) proposed that the modulation of base-word frequency effects and the latency advantage/ disadvantage for pseudohomophones in the naming task reflect the degree to which readers strategically adjust how information from the lexicons is used to create pronunciations. Under the general activation strategy (GAS), lexical entries that are similar to the target string in both spelling and sound contribute to naming. Under the specific activation strategy (SAS), the letter string's pronunciation is driven largely by its matching representation in the orthographic and/or phonological lexicon(s). According to this account, the naming advantage for pseudohomophones in a list that also contains control nonwords reflects the operation of the GAS such that the general activation of entries in the lexicon aids in the formation of a pronunciation. As a consequence of the lexicons' activity, pseudohomophones are pronounced more quickly than control nonwords. The activation of the lexicons does not lead to a naming advantage for pseudohomophones based on high-frequency words over those based on low-frequency words. The baseword frequency effect is absent because the lexicon's activity is diffused over the entire content of the lexicon. A frequency effect will occur when the model accesses the base word under the SAS.

In a pure list of pseudohomophones, every item has a matching code in the phonological lexicon. Readers use the pseudohomophone's corresponding base word to help construct a response - that is, they activate the SAS. Because fewer lexical entries end up being used to generate a pronunciation under the SAS than under the GAS, the model requires more time (measured in cycles) to bring phonemes in the phoneme system to criterion under the SAS than under the GAS. Given that the SAS entails the use of single lexical entries to construct a pronunciation, pseudohomophones based on high-frequency words are named more quickly than those based on low-frequency words.

In their demonstrations that the different strategies could account for human data patterns, Reynolds and Besner (2005) set out to simulate pseudohomophone reading by varying a single parameter of the DRC model. They selected the parameter that governs the inhibition from letter nodes to orthographic entries in the lexicon. A side effect of this change, however, was an increase in the model's tendency to report a nonword as a word (i.e., lexical capture). To counteract lexical capture, the simulations included a constant change in three other parameters. Lateral inhibition among entries in the two lexicons was set to 0 , and the excitatory connections between entries in the phonological lexicon down to the phonemes at the output layer were reduced (from .14 to .014).

To simulate the GAS, inhibitory weights between letter nodes and entries in the orthographic lexicon were reduced from -.435 to -.25 . This reduction allows entries in the orthographic and phonological lexicons to contribute to the pronunciation of a nonword by de-emphasizing the impact of mismatches between the letters of a target letter string and the lexical entries that are activated in response to them. To simulate the SAS, the letter-to-orthographic inhibition parameter was maintained at its default value of - .435. Reynolds and Besner (2005) showed that with the SAS and GAS parameter settings, the DRC model did a good job of simulating the difference in performance on naming words and performance on naming pseudohomophones, as well as the base-word frequency effects exhibited by readers.

Reynolds and Besner (2005) also found that when subjects named the items in a pure list of pseudohomophones after naming items in a list of control nonwords, the pseudohomophone disadvantage was attenuated relative to when pseudohomophones were named first. As well, the effect of base-word frequency on pseudohomophone naming was attenuated when the pseudohomophones were presented after a list of control nonwords. They claimed that another strategy - the intermediate activation strategy (IAS) - was responsible for such context effects. Specifically, they suggested that the effect of list context occurs because readers adopt a strategy that has properties of both the SAS and the GAS. To simulate the IAS, the inhibitory weights connecting letters to entries in the orthographic lexicon were set to the value of -.385 (between the values set for the SAS [-.435] and the GAS [-.25]). Under the new parameter setting, the DRC model again did an adequate job of accounting for the interaction among context, stimulus type (nonword vs. pseudohomophone), and base-word frequency.

\section{Controlling the GAS}

We concur with Reynolds and Besner (2005) that the range of pseudohomophone effects may be explained within the context of strategic control's modulation of the influence of general or specific word knowledge. In this section, we explore a way of controlling the specificity of the lexicons' involvement in pronunciation without adjusting the strength of the connections between objects in memory. Our motivation for extending Reynolds and Besner's examination was driven by considering how cognitive penetrability constrains the strategic control of reading. What mechanisms are under the reader's control? In the interactive activation framework (McClelland \& Rumelhart, 1981) used by the DRC model, excitatory and 
inhibitory connections between nodes are properties of the data known to the system. Because interconnections represent data, it is unclear how the reader would be able to change their strengths to exploit different strategies.

Reynolds and Besner (2005) were careful not to commit to the methods they used to induce the specific, intermediate, and general activation strategies in the DRC model. The point of their demonstration was to provide an existence proof that different parameter sets within the DRC model control how general and specific information from the lexicon affects pseudohomophone naming.

Our starting point in extending their argument is the recent account of strategic reading processes proposed by Lupker, Brown, and Colombo (1997). The hypothesis is that readers "establish a flexible deadline for when articulation should start" (Lupker et al., 1997, p. 582). Where the deadline is placed depends on how difficult the letter string is to process or how much time the reader believes is appropriate to process the stimulus. In general, if the letter string is difficult to process (e.g., if it is a low-frequency irregular word such as chef), the reader will wait longer before beginning articulation than if the letter string is easy to process (e.g., if it is a high-frequency regular word such as $d o g$ ). The account also predicts that when easy and difficult stimuli are mixed together in a list, the deadline is placed somewhere between the locations for a pure list of easy items and a pure list of difficult items.

The DRC model has one parameter (referred to by Coltheart et al., 2001, as the reading-aloud criterion) that controls the activation that must be reached in the phonemic system before its information is sent on for articulation. Over successive processing cycles, phonemes in the phoneme system are scanned to determine whether or not a pronunciation has been settled upon. The model has settled on a pronunciation when a set of phonemes in the phoneme system has reached a criterion amount of activation. A large value of the parameter such as .90 reflects leisurely reading; lower values reflect speeded reading. ${ }^{1}$ In the DRC simulations reported by Coltheart et al., the parameter was set to .43 .

We postulate that the specific and general activation strategies reflect different values of the reading-aloud criterion and that readers adjust its value in response to the stimuli in a list. When the reading-aloud criterion is set low (e.g., .35), the activation in the phoneme system will rise to criterion more quickly than it will when the reading-aloud criterion is left at its default value of .43. Likewise, increasing the criterion (e.g., .70) above the default value of .43 will slow naming latencies.

Changing the criterion affects more than the system's naming latency; it also influences the degree to which the lexicons contribute to the pronunciation of a letter string. Within each lexicon, entries (represented as nodes) possess an inhibitory connection to every other node in the lexicon. When the DRC model is presented with a letter string that matches or resembles a word in the lexicon, the activation of the word's node is increased. The more the word's node is activated in response to the letter string, the more the node inhibits other word nodes that do not match the letter string.
On successive processing cycles, the system moves closer and closer to converging on a single lexical match to the target string. Hence, when set to a low value, the reading-aloud criterion will lead to short naming latencies with only general lexical information contributing to a pronunciation (GAS). As the criterion is raised, the increased processing cycles lead to an increase in the degree to which matching lexical entries contribute to a pronunciation (SAS). That is, when the reading-aloud parameter is set high, lexical activation leading to a pronunciation becomes increasingly less diffuse. As a consequence, the strength of the relationship between naming latency and the printed frequency of a word (or the base word of a pseudohomophone) will increase.

\section{A Simulation}

To explore this idea, we ran two versions of the DRC model on the 90 pseudohomophones (45 based on lowfrequency words and 45 based on high-frequency words) and 90 control nonwords listed in Appendix A of Reynolds and Besner (2005). The first version, which we will refer to as DRC 1.0, is the version reported by Coltheart et al. (2001) and tested by Reynolds and Besner. The second version-DRC 1.1.4 (see www.maccs.mq.edu.au/ $\sim$ Ssaunder/DRC/) - is the most recent version. ${ }^{2}$ We ran the models on the items six times with the reading-aloud parameter set to $.25, .35, .43$ (the default value used by DRC 1.0), .50, .55, and .70. All the other parameters were held at their respective default values. In what follows, we examine the influence that changing the parameter value has on the strength of the base-word frequency effect in pseudohomophone naming, context effects, and whether pseudohomophones show an advantage or a disadvantage relative to nonword controls.

Figure 1 shows the models' correct naming latencies as a function of the value of the reading-aloud parameter. The figure shows clearly that naming latencies increase with the parameter values. The increase reflects the additional number of processing cycles required for the phonemes in the phoneme system to reach criterion. Also shown in Figure 1 is a naming advantage for pseudohomophones that increases with the reading-aloud parameter.

As was mentioned above, increasing the reading-aloud parameter increases the extent to which the different lexicons contribute to the generation of a pronunciation (mirroring the distinction between the GAS and the SAS). During the transition from general to specific lexical involvement, pseudohomophone and nonword pronunciations are impacted by increasingly fewer lexical entries. As the lexical involvement in the pronunciation increases, the impact on control nonwords becomes increasingly negative. Nonwords have no status in either lexicon; hence, as the model moves to greater specific lexical involvement, it begins to experience lexical capture. With lexical capture comes longer naming times and an increased tendency to make lexicalization errors (e.g., pronouncing the nonword derd as dead). In the simulations we ran, only DRC 1.0 made any pronunciation errors. At reading-aloud criterion values of $.5, .6$, and .7 , the model had error rates of .01, .02, and .04 , respectively. All the errors were lexicalizations. 


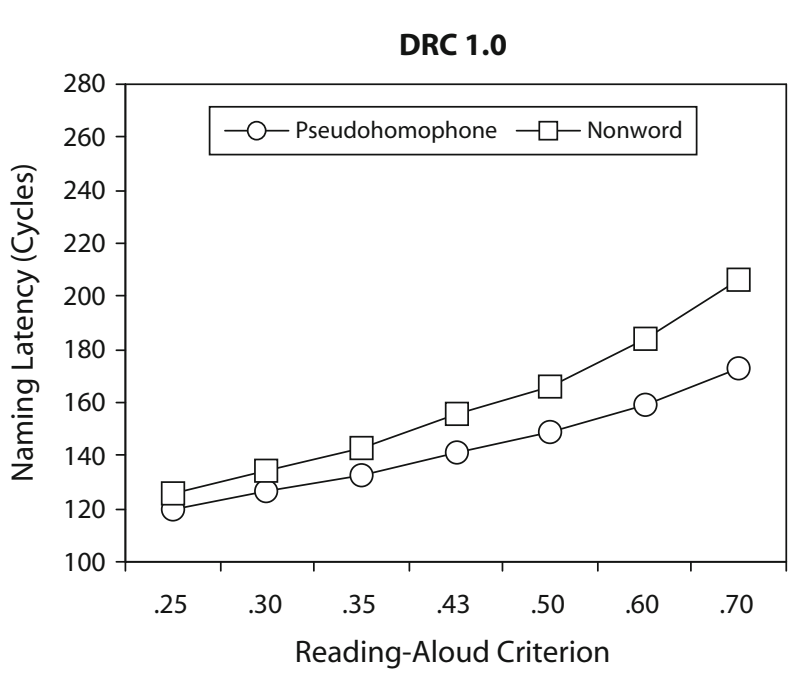

DRC 1.1.4

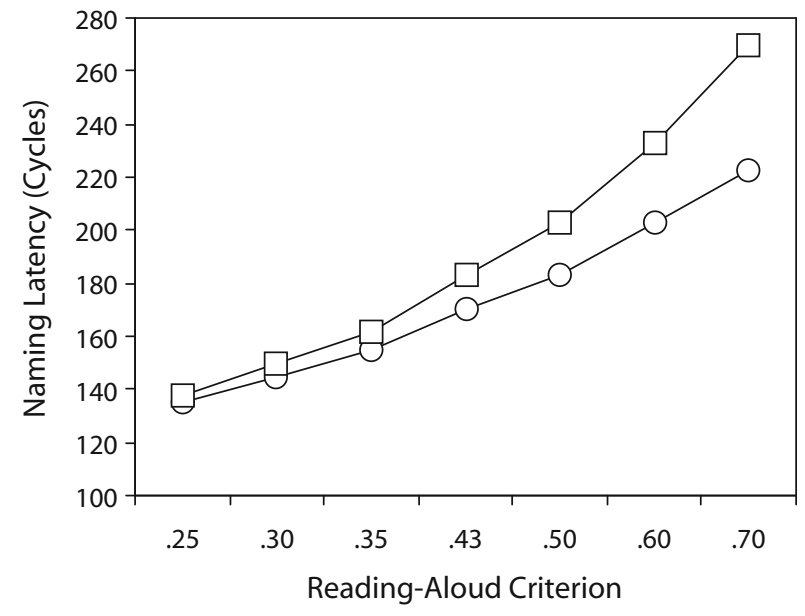

Figure 1. Naming latencies from DRC 1.0 (top panel) and DRC 1.1.4 (bottom panel) for pseudohomophones and nonword controls as a function of reading-aloud criterion value.

Additional evidence for the notion that the readingaloud parameter may govern lexical contributions is found in the model's sensitivity to base-word frequency when pseudohomophones are named. Figure 2 shows the magnitude of the naming advantage for pseudohomophones based on high-frequency words over ones based on low-frequency words as a function of where the reading-aloud parameter is placed. The figure shows that, for both versions of the DRC model, naming latencies for pseudohomophones become increasingly sensitive to base-word frequency as the parameter is increased.

The reading-aloud criterion parameter determines, albeit indirectly, the degree to which the DRC model moves from general to specific lexical information to help build a pronunciation. In previous work reporting simulations using the DRC model (e.g., Coltheart et al., 2001), the reading-aloud criterion has been set at .43. This value was chosen because it allowed the model to account for several important phenomena in the reading literature while maintaining an acceptable error rate for words and nonwords.
We acknowledge that fixing parameters is important in accounting for differences in performance that arise from changing classes of stimuli. However, fixed values preclude the model from explaining phenomena that reflect strategic changes to parameters that occur in response to instructions or to the structure of stimulus lists.

Assuming that placement of the criterion is under strategic control, how does the reader decide on an appropriate value? We believe that readers place the criterion at a value that they deem appropriate given their instructions and what they know about the stimuli. For example, when subjects are told that a list contains nonwords that sound like words, the best strategy might be to raise the readingaloud parameter from the default value to increase the impact that matching lexical entries have on pronunciation. On the other hand, if subjects are told that a list contains nonwords that do not sound like words, reducing the parameter from its default may be an effective strategy for avoiding lexical capture without sacrificing accuracy. When the two classes of stimuli are mixed together, the best place for the criterion is somewhere between the two extremes - akin to the strategy Reynolds and Besner (2005) referred to as the IAS.

Table 1 presents naming latencies for control nonwords and pseudohomophones from DRC 1.0 and DRC 1.1.4 using reading-aloud criterion settings to simulate pure and mixed lists. For pure lists, the values were set at .43 and .70 for nonwords and pseudohomophones, respectively. To simulate a mixed list, the items were named with the reading-aloud parameter set at .50. As is clear in the table, both versions of the DRC model show a definite latency disadvantage for pseudohomophones in pure lists but an advantage in mixed lists.

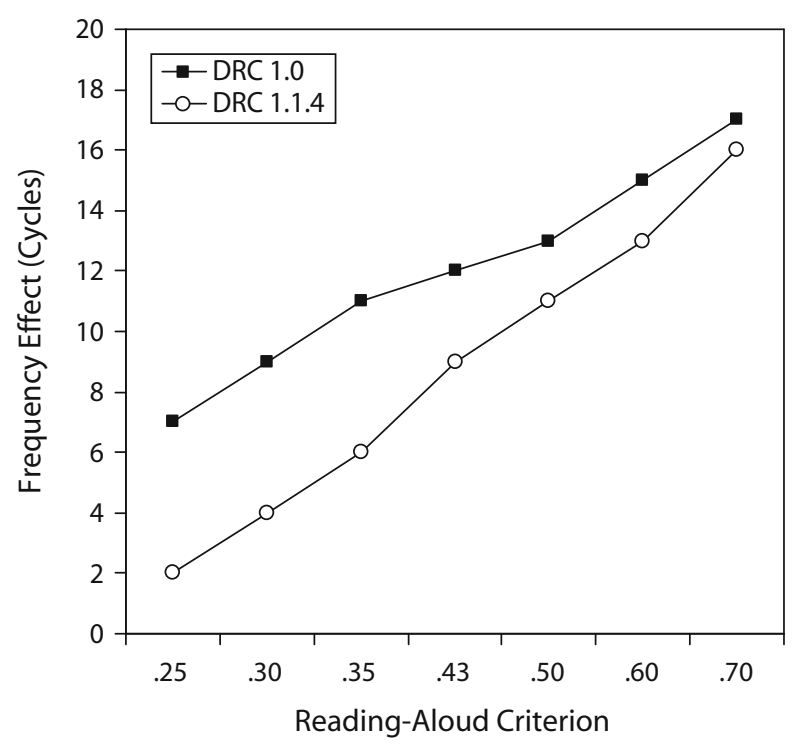

Figure 2. Differences between the naming latency of pseudohomophones derived from high-frequency base words and that of pseudohomophones derived from low-frequency base words as a function of the reading-aloud criterion value. 
Table 1

Naming Latencies (in Processing Cycles) for Pseudohomophones and Nonwords in DRC 1.0 and DRC 1.1.4

\begin{tabular}{|c|c|c|c|c|c|}
\hline \multirow[b]{3}{*}{ Model } & \multirow[b]{3}{*}{ List Type } & \multicolumn{4}{|c|}{ Stimulus Type } \\
\hline & & \multicolumn{2}{|c|}{ Pseudohomophone } & \multicolumn{2}{|c|}{ Nonword } \\
\hline & & RAP & Cycles & RAP & Cycles \\
\hline \multirow[t]{2}{*}{ DRC 1.0} & pure & .70 & 173 & .43 & 156 \\
\hline & mixed & .50 & 148 & .50 & 166 \\
\hline \multirow[t]{2}{*}{ DRC 1.1 .4} & pure & .70 & 223 & .43 & 183 \\
\hline & mixed & .50 & 184 & .50 & 203 \\
\hline
\end{tabular}

Note-Pure and mixed lists were simulated by adjusting the readingaloud parameter (RAP).

Reynolds and Besner (2005) found that the disadvantage for pseudohomophones in a pure list and the base-word frequency effect were attenuated when subjects named items in a list of control nonwords before naming those in a list of pseudohomophones. We interpret the context effect as reflecting how the reader adjusts the reading-aloud parameter from the initial value set for the first list to the new value in the second. We propose further that the impact of context on naming reflects a differential carry-over effect from the first list to the second. When pseudohomophones are named first and subjects are told that each item sounds like a word, the criterion is set high so that lexical entries play a role in driving a pronunciation (i.e., the GAS). We set the criterion at .70 in both models to encourage lexical capture. In a manner similar to a verification process (Marmurek \& Kwantes, 1996), the parameter controls the degree to which feedback from the lexicons is used to help in constructing a pronunciation.

After subjects finish a list of pseudohomophones, they are told that the items in the second new list do not sound like words. Subjects begin the task with the second list with substantial practice at reading nonwords, and knowing that none of the stimuli sound like words means they can lower the criterion drastically without compromising accuracy. For the simulation reported here, we dropped the criterion to .43 in DRC 1.0 and to .50 in DRC 1.1.4. ${ }^{3}$

When nonwords are shown first, subjects are unpracticed and set the reading-aloud parameter to a medium level. We used values of .35 in DRC 1.0 and .43 in DRC 1.1.4 to reflect the criterion's placement. After the nonword list is read, the subject is a practiced nonword reader. When the pseudohomophones of the second list are presented, the subject increases the criterion to increase the lexicons' roles in creating a pronunciation. However, because subjects know the pseudohomophones are nonwords and are practiced at reading them, the criterion does not need to be increased by a large amount to successfully accomplish the task (we increased it to .43 in DRC 1.0 and to .50 in DRC 1.1.4).

The three panels of Figure 3 show the DRC performance in comparison with that of human subjects reported by Reynolds and Besner (2005). There is a close correspondence among the patterns exhibited by humans and by the two versions of the DRC model. Furthermore, recall that the DRC model's sensitivity to word frequency increases as the reading-aloud parameter is raised. Con- sistent with this fact, the latency advantage for highfrequency over low-frequency words is larger when pseudohomophones are named first $(M=17$ cycles for DRC

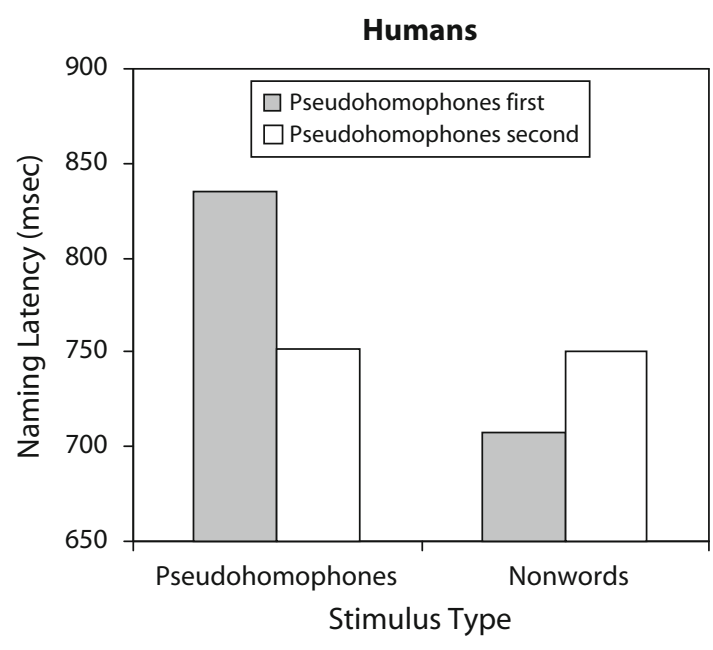

DRC 1.0

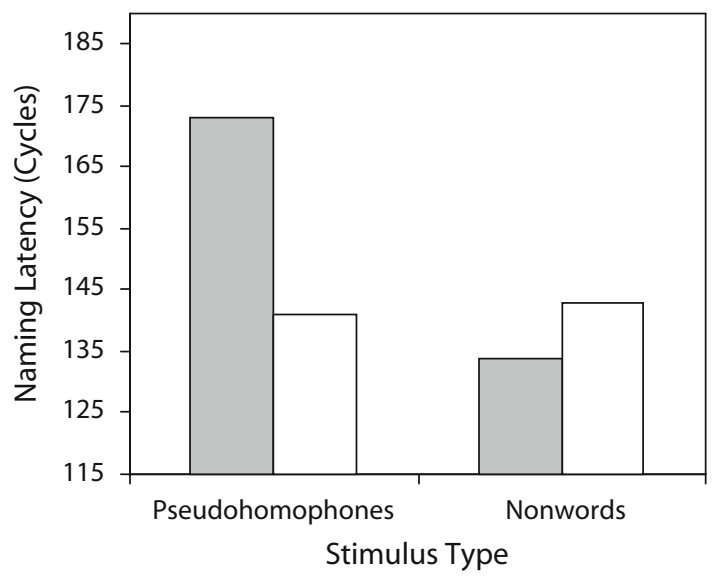

DRC 1.1.4

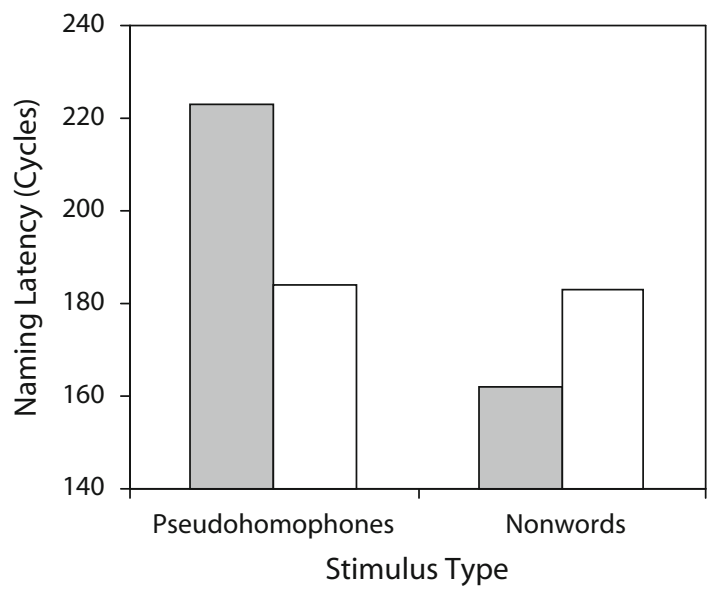

Figure 3. Naming latencies for humans (top panel, taken from Reynolds \& Besner, 2005), DRC 1.0 (middle panel), and DRC 1.1.4 (bottom panel) for presentation of pseudohomophones and of nonwords in the first list and in the second list. 
1.0 , and $M=16$ cycles for DRC 1.1.4) than when named following a list of control nonwords $(M=12$ cycles for DRC 1.0 and $M=11$ cycles for DRC 1.1.4).

\section{Conclusion}

We showed that varying the reading-aloud parameter in the DRC model without concomitant changes to any other default values can account for the strategic modulation of base-word frequency, the pseudohomophone advantage/ disadvantage, and list order effects when pseudohomophones are read aloud. The reading-aloud parameter controls how active phonemes at the output layer of the model must become before articulation begins. We concur with Reynolds and Besner (2005) that the critical determinant of the pseudohomophone effects is the degree to which entries in the lexicons drive pronunciations in response to the stimuli that readers are asked to process. We propose that the locus of the control is not found in the data of the lexicon (which may not be cognitively penetrable), but, rather, that the locus of strategic lexical control is an adjustment on how much evidence readers require in the phoneme system before articulation begins. That adjustment indirectly dictates how much of a role the lexicons play in the construction of a pronunciation.

Our proposal for strategic control in the naming task is similar to that of Lupker et al. (1997), who argued that readers adjust a deadline for beginning articulation in response to how difficult a letter string is to process. Our explanation for the naming task implicates control over a criterion for evidence rather than one for time. However, because the DRC model builds evidence for a pronunciation over time, the distinction is blurred in that model. Further research is needed to explore the extent to which strategic adjustment of the DRC model's reading-aloud parameter explains the strategic effects reported by Lupker et al.

\section{AUTHOR NOTE}

This research was supported by a Natural Sciences and Engineering Research Council of Canada grant to H.H.C.M. We are grateful to Derek Besner and Stephen Goldinger for useful comments on earlier drafts of this article. Correspondence concerning this article should be addressed to H. H. C. Marmurek, Department of Psychology, University of Guelph, Guelph, ON, N1G 2W1 Canada (e-mail: marmurek@ psy.uoguelph.ca).

\section{REFERENCES}

Borowsky, R., Owen, W. J., \& Masson, M. E. J. (2002). Diagnostics of phonological lexical processing: Pseudohomophone naming advantages, disadvantages, and base-word frequency effects. Memory \& Cognition, 30, 969-987.

Coltheart, M., Rastle, K., Perry, C., Langdon, R., \& Ziegler, J. (2001). DRC: A dual route cascaded model of visual word recognition and reading aloud. Psychological Review, 108, 204-256.

Grainger, J., Spinelli, E., \& Ferrand, L. (2000). Effects of baseword frequency and orthographic neighborhood size in pseudohomophone naming. Journal of Memory \& Language, 42, 88-102.

Herdman, C. M., LeFevre, J.-A., \& Greenham, S. L. (1996). Baseword frequency and pseudohomophone naming. Quarterly Journal of Experimental Psychology, 49A, 1044-1061.

LupKer, S. J., Brown, P., \& Colombo, L. (1997). Strategic control in a naming task: Changing routes or changing deadlines? Journal of Experimental Psychology: Learning, Memory, \& Cognition, 23, 570-590.

Marmurek, H. H. C., \& Kwantes, P. J. (1996). Reading words and wirds: Phonology and lexical access. Quarterly Journal of Experimental Psychology, 49A, 696-714.

MCCANN, R. S., \& BESNER, D. (1987). Reading pseudohomophones: Implications for models of pronunciation assembly and the locus of word-frequency effects in naming. Journal of Experimental Psychology: Human Perception \& Performance, 13, 14-24.

McClelland, J. L., \& Rumelhart, D. E. (1981). An interactive activation model of context effects in letter perception: I. An account of basic findings. Psychological Review, 88, 375-407.

Reynolds, M., \& Besner, D. (2005). Basic processes in reading: A critical review of pseudohomophone effects in reading aloud and a new computational account. Psychonomic Bulletin \& Review, 12, 622-646.

TAFT, M., \& Russell, B. (1992). Pseudohomophone naming and the word frequency effect. Quarterly Journal of Experimental Psychology, 45A, 51-71.

\section{NOTES}

1. We thank Max Coltheart for verifying the role played by the readingaloud parameter.

2. We thank Derek Besner for making us aware of the new version of the DRC model. Unlike the original DRC, in which the reading-aloud parameter is set to a default that reflects speeded reading (.43), the default value in DRC 1.1.4 is set to .90 to reflect leisurely reading.

3. There are some differences in how the two versions of the DRC model are parameterized. As a consequence, the two versions sometimes require different values of the reading-aloud parameter to capture the phenomena reported here. Although the numbers may differ, the qualitative changes in the parameters required to capture the effects were constant across versions of the model.

(Manuscript received March 8, 2006; revision accepted for publication May 18, 2006.) 